\title{
The Impact of Connecting Distributed Generation to the Distribution System
}

\begin{abstract}
E. V. Mgaya, Z. Müller
This paper deals with the general problem of utilizing of renewable energy sources to generate electric energy. Recent advances in renewable energy power generation technologies, e.g., wind and photovoltaic (PV) technologies, have led to increased interest in the application of these generation devices as distributed generation $(D G)$ units. This paper presents the results of an investigation into possible improvements in the system voltage profile and reduction of system losses when adding wind power DG (wind-DG) to a distribution system. Simulation results are given for a case study, and these show that properly sized wind DGs, placed at carefully selected sites near key distribution substations, could be very effective in improving the distribution system voltage profile and reducing power losses, and hence could improve the effective capacity of the system.
\end{abstract}

Keywords: renewable energy sources, distributed generators, wind power plant, power systems disturbances, flicker and steady-state stability.

\section{Introduction}

One of the present-day tasks for power engineers is to generate electricity from renewable energy sources (RES). Increasing energy consumption and diminishing conventional sources have made people think of utilizing renewable sources in conventional power systems. The use of renewable sources of energy promotes sustainable living and, with the exception of biomass combustion, it is virtually pollution free. RES are economically feasible in small-scale applications in remote locations (away from the grid areas) or in large-scale applications in areas where the resource is abundant and can be harnessed by giant conversion systems. Distributed generators can be connected to medium voltage (MV) or low voltage (LV) distribution systems. Two basic methods are used to supply electricity to a certain area. The first is to transfer the required energy using conventional power systems, and the second one is to produce it directly at the location where it will be consumed. A combination of the two methods can be used. The first method is frequently used, but small power generators located close to energy consumers are very interesting. This paper will draw attention to the impacts of connecting wind power plants to the existing system.

\section{Wind power plants}

Wind is formed by changes in pressure between differently heated areas of air in the earth's atmosphere. The velocity of the wind, which is the most important parameter for exploitation of wind energy, is proportional to the amount of pressure difference. The minimum economical limit for exploitation of the wind energy all over the world is $5 \mathrm{~m} / \mathrm{s}$. The upper wind speed limit is considered to be about $25 \mathrm{~m} / \mathrm{s}$. Higher wind speeds are dangerous because they may damage the equipment of the power plant.

\section{Power system disturbances}

Electrical energy is a commodity in the market, and it is necessary to set certain rules for rating its quality. In earlier times, electricity was rated only according to voltage and frequency stability; however the use of more advanced appliances and devices with non-linear or changing characteristics has had an adverse impact on electricity distribution systems. The adverse impacts can cause disturbances to other appliances and devices in that system. The disturbances are categorized according to the frequency (power systems, acoustic, radio). This paper deals with power system disturbances due to higher harmonics, interharmonics, voltage fluctuation, flicker and voltage imbalance.

\subsection{Higher harmonics}

Higher harmonics are sinusoidal waves of voltages and currents with frequency integer multiples of $50 \mathrm{~Hz}$. The most common sources of higher harmonics are devices with a non-sinusoidal current. If we want to maintain a voltage balance between a system and a consumer that affects the system adversely with higher harmonics, a higher harmonic current must flow through a part of the circuit. This current creates voltage drops on the inductances and resistances of the circuit. These higher harmonic voltages are superimposed on the sinusoidal voltage of the system frequency. These currents and voltages cause additional stress to other devices connected to the system. If the stress exceeds the resistance level, the following may occur:

- Reduction of lifetime,

- early failures of capacitors and motors because of overloading,

- function failures of electrical devices,

- wrong protection function,

- malfunction of ripple control receivers,

- negative effect on arc extinction.

The higher harmonic voltage should not exceed certain limits, in order to avoid the phenomena mentioned above. Higher harmonic currents are influenced by electrodynamic phenomena, e.g., skin effect and proximity effect. These phenomena cause an increase in resistances and active losses.

Higher harmonic currents and voltages form additional losses, which are included in the "deformational power". The apparent power $S$ can be expressed in terms of active power $P$, reactive power $Q$ and distortive power $D$ :

$$
S^{2}=P^{2}+Q^{2}+D^{2} \text {. }
$$


Deformational power influences the "true power factor" $\cos \varphi$. An electronic energy meter can measure this true power factor, while classical inductive energy meters measure only the first harmonic. Fig. 1 shows the geometrical relationship between apparent, active, reactive and distortive power.

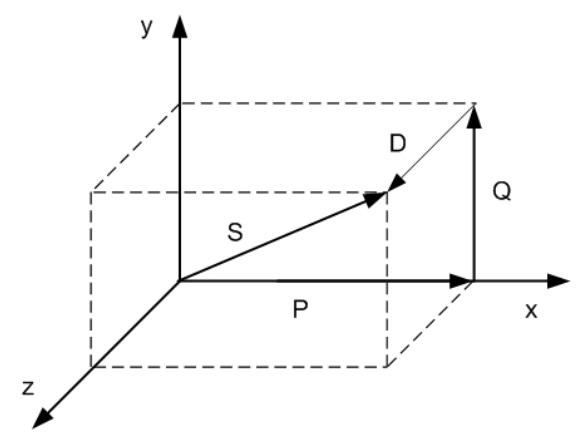

Fig. 1: Geometrical relationship of powers

\subsection{Interharmonics}

Interharmonics are all sinusoidal waves of voltage and current having a frequency that is a non-integer multiple of the system frequency. Interharmonic voltages create an additional distortion of the voltage waves and are not periodic with the system frequency. Interharmonic sources are converters with direct inter-circuit, straight frequency converters, rectifying cascades and electronic devices working in cycles (heat devices, machines with changing load torque). Interharmonics have a negative influence on the ripple control signal.

Interharmonics are the main cause of flicker and disturbance of ripple control signal receivers. They can be reduced by smoothing the direct inter-circuit currents at frequency converters or by choosing a suitable point of common coupling (PCC) at places with higher short-circuit power.

\subsection{Voltage fluctuation}

Voltage fluctuation is a disturbance phenomenon that arises during the operation of electrical devices with a varying load. These changes cause different voltage drops on impedances, resulting in voltage changes at places of consumption. The changes in voltage cause changes in light flux (flicker) at light sources, mainly bulbs. This voltage fluctuation can be periodic or stochastic.

The sources of voltage fluctuations can be controlled converters, welding machine sets, electrical resistors and arc furnaces, all consumers with pulse consumption, large load switching or asynchronous generators in wind power plants.

Voltage fluctuation can be reduced by connecting a large consumer to a system with a higher short-circuit power, or by strengthening the system.

In order to achieve higher short-circuit power (MVA) the following can be done:

- Increase the installed power of the supply transformer,

- connect a new generator, a synchronous compensator,

- decrease the supply line impedance by means of serial compensation,

- connect to a higher voltage level.

\section{Conditions for connecting distributed generators to a distribution system}

The possibility of connecting distributed generators to a distribution system is judged according to their negative impacts on the distribution system. Our judgment is based on the system impedance at the point of common coupling (PCC), short-circuit power, resonance, connected power and the type of distributed generators (wind, small hydro, etc.)

The following subsections refer to the requirements of the Czech (European) technical standards for connecting distributed generators to the distribution network.

\subsection{Size of the connected power}

The size of the power to be connected to the existing distribution system depends upon the voltage level of that system. If the power is to be connected to LV systems then the total rated power of the distributed generators to be connected should not exceed $10 \%$ of the distribution transformer rated power. And if the power is to be connected to the MV system then the total rated power of the distributed generators to be connected should not be higher than $10 \%$ of the supply transformer 110/22 kV rated power.

\subsection{Increase in voltage}

The voltage increase in the MV systems after connecting the distributed generators at the point of common coupling (PCC) should in the most unfavorable case not exceed a value of $2 \%$ in comparison with the voltage before their connection, and distributed generators connected to the LV systems should not increase the voltage by more than $3 \%$.

\subsection{Voltage changes during switching}

Connecting and disconnecting individual generators causes voltage changes at PCC. In the MV system, these changes do not cause undue negative impacts provided that the highest voltage change at PCC does exceed $2 \%$, and for LV systems the voltage change limit is $3 \%$.

\subsection{Long-time flicker}

When analysing one or many distributed generators at PCC, it is important to consider the voltage fluctuation that causes flicker. At PCC it is therefore necessary to keep to the limits for the long-time flicker perception rate $P_{l t}$ and for the long-time flicker factor $A_{l t}$ as follows:

$$
\begin{aligned}
& P_{l t} \leq 0.46, \\
& A_{l t} \leq 0.1 .
\end{aligned}
$$

The long-time flicker perception rate $P_{l t}$ is determined using the following equation:

$$
P_{l t}=c \frac{S_{n E}}{S_{k V}}
$$

where:

$\begin{array}{ll}c & \text { is flicker factor [-], } \\ S_{n E} & \text { is load rated power [MVA], } \\ S_{k V} & \text { is system short-circuit power [MVA]. }\end{array}$


If the producer includes more than one generating unit, $P_{l t}$ must be calculated separately for each device and the resulting flicker value $P_{\text {ltres }}$ at PCC is then determined:

$$
P_{\text {lt res }}=\sqrt{\sum_{i} P_{l t i}^{2}}
$$

\subsection{Higher harmonics in MV systems}

The allowable higher harmonic currents of the individual equipment connected at PCC can be computed using the following equation:

$$
I_{v p r i}=I_{v p r} \frac{S_{A}}{S_{A V}}=i_{v p r} \cdot S_{k V} \cdot \frac{S_{A}}{S_{A V}},
$$

where:

$S_{A} \quad$ is single device apparent power [MVA],

$S_{A V}$ is total connected power [MVA],

$i_{v p r} \quad$ is relative current [A/MVA],

$S_{k V}$ is distribution system short-circuit power [MVA],

$I_{v p r} \quad$ is higher harmonic allowable current [A].

If the allowable harmonic currents are exceeded, then connection at PCC is not possible.

\section{A study of the wind power plant connection}

Before the wind power plant was connected, the distribution network was fed at two points from the $110 / 22 \mathrm{kV}$ system through transformer T1 with $25 \mathrm{MVA}$ and transformer T2 with 25 MVA. The short-circuit power at the first and second point is 1200 MVA and 1000 MVA respectively. Now another supply from the wind power plant is connected to the distribution network through transformer T3 rated 8MVA, $22 / 0.7 \mathrm{kV}$.

The $22 \mathrm{kV}$ distribution network consists of an overhead line 95AlFe6 with a maximum current carrying capacity of $289 \mathrm{~A}$, and the total length of the line is about $40 \mathrm{~km}$. The distribution network is connected to the wind power plant through a 70AlFe6 line with maximum current carrying capacity of $225 \mathrm{~A}$ and about $2 \mathrm{~km}$ in length.

The wind power plant consists of four synchronous Vestas V80-1800 kW type generators with nominal voltage of $690 \mathrm{~V}$, nominal current of $1390 \mathrm{~A}$ and power factor range from 0.90 (inductive) to 0.98 (capacitive).

The system steady-state calculations were performed using the EGC VLIVY 4.2 and DAISY PAS Off - Line V 3.5 programmes. The calculations were performed for all generator combinations (without connecting the generators to the sequential connection of all four generators) and further for different power factors in the range 0.98 cap -0.90 ind with a step of 0.02 . The calculation was performed for minimum line loading of $66 \mathrm{~A}$ and maximum line loading of $108 \mathrm{~A}$.

\subsection{Line losses}

The calculations of the line losses were performed during the network steady state calculation. The losses in Fig. 2 were calculated for cases when all loads are supplied from substations T1, T2 and the wind power plant contributes only $60 \%$ of its rated power. It is seen during the analysis that the line losses are lower when the generators do not contribute to the network and it is fed from both substations than when the generators are connected sequentially to the system. Generally, the number of connected generators influences whether the losses are higher at minimum or maximum line loading. Relatively high line losses occur when the generators work in an overexcited state with the power factor $\cos \varphi=0.98$ cap. So in order to operate this network with minimum losses, we have to regulate the power of the wind power plant according to the immediate consumption in the network.

\subsection{Voltage profiles}

The voltage profiles were constructed at $60 \%$ generator loading. The profiles are shown in Fig. 3. Only a small change in voltages occurs at substations T1 and T2 after connecting

Losses on all network $60 \%$ power of generators

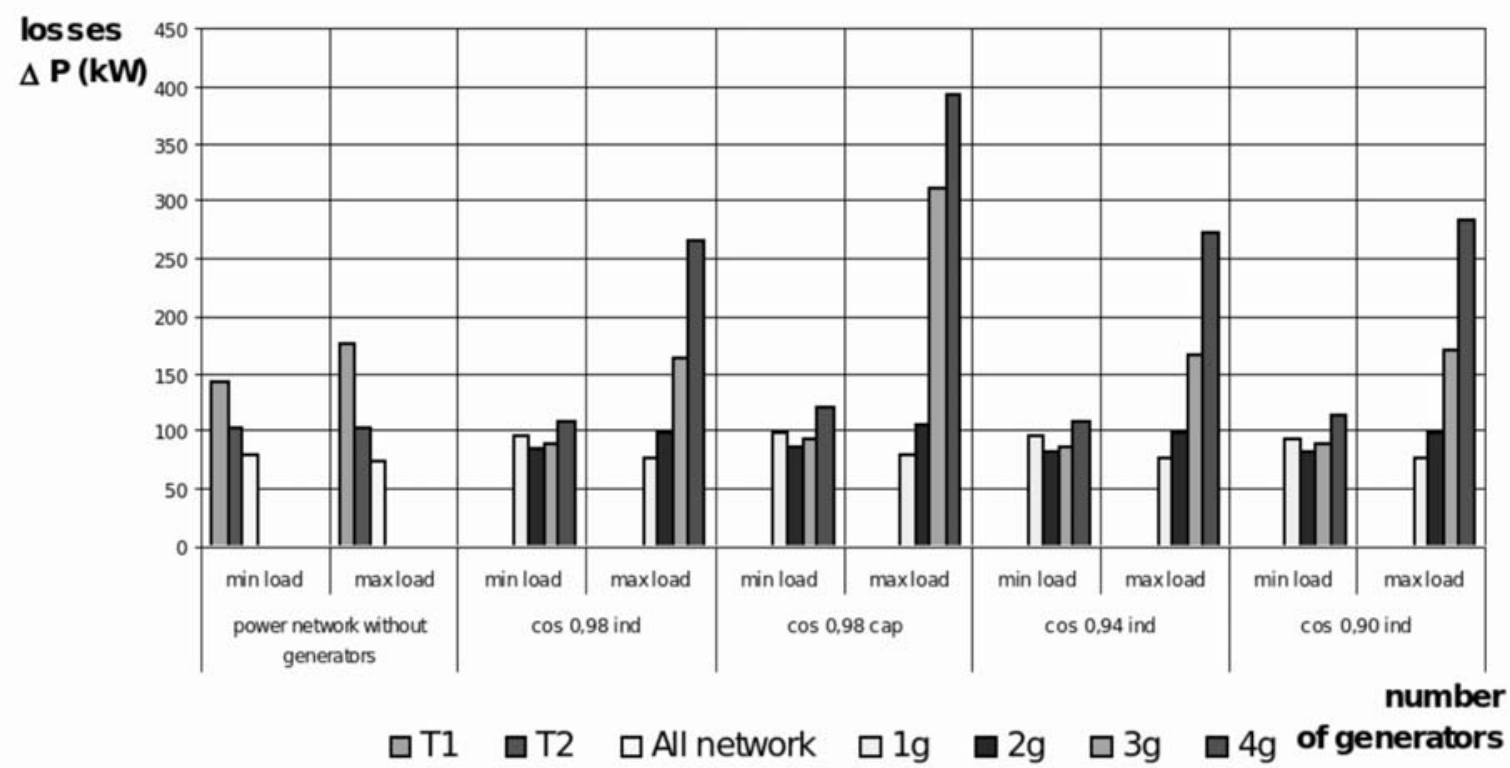

Fig. 2: Line losses when the network is supplied by substations T1, T2 and at $60 \%$ generator loading 


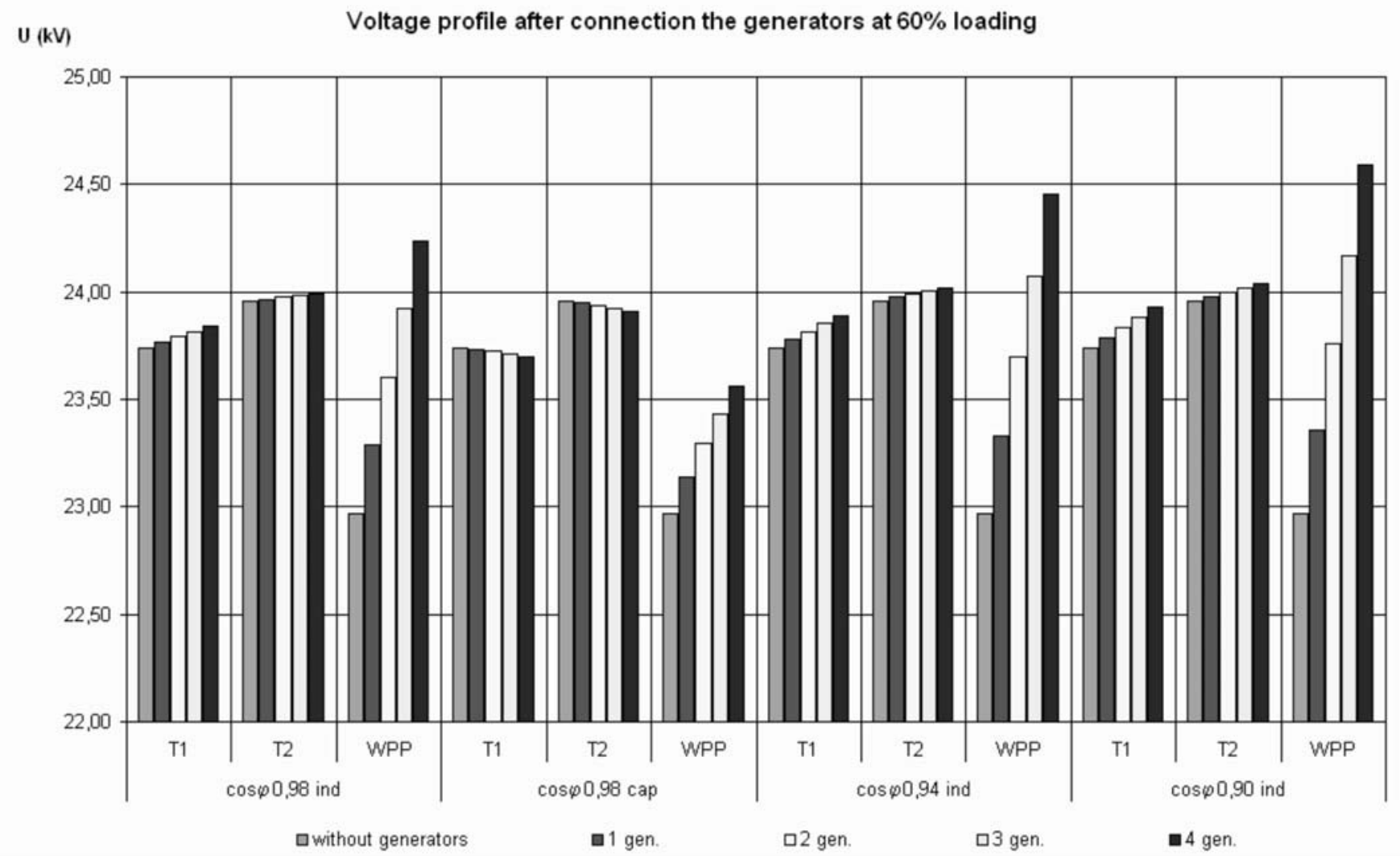

Fig. 3: Voltage profile after connecting the generators at $60 \%$ loading

the generators to the system. This is due to the higher short-circuit power of the system where the substations are connected than that of the system where the wind power plant works. In MV systems according to the technical standards of the distribution utility, the maximum voltage difference must not exceed $2 \%$ of the original voltage profile. This criterion is fulfilled only for some cases of generator connection. The size of the voltage difference depends on the number of generators connected and the power factor.

\subsection{Ripple control signal (RCS) damping}

Since electrical generators can unfavorably influence the level of the Ripple Control Signal (RCS), this level must not drop by more than $10 \%$ to $20 \%$ below the required level. When the four synchronous Vestas V80 - $1800 \mathrm{~kW}$ machines are connected at the point of common coupling (PCC) $22 \mathrm{kV}$, the RCS damping is lowered by additional supporting impedance.

\subsection{Flicker}

Another qualitative parameter for judging the connection of a wind power plant is the flicker value caused by voltage variation. The flicker coefficient $P_{l t}$ at the point of common coupling (PCC) must not exceed a value of 0.46 . It was proved by calculation that a value 0.46 of flicker coefficient $P_{l t}$ was not reached.

\subsection{Contributions of harmonic currents}

The checks on the allowable size of the harmonic and interharmonic currents that reached the network from the wind power plant when it was connected to the network proved that the allowable limits were not exceeded.

\section{Stability of the system with the wind power plant}

The existing technical rules commonly used by distributors for connecting generators to the network are as follows:

- Increase in voltage,

- islanded operation (and anti-islanded protection),

- increase in short circuit level,

- power quality (harmonics, voltage drops, unbalanced voltage etc.).

In some countries (France, Italy) simple rules are applied which define the voltage level to which a distributed generator may be connected, depending on its rated power output.

It appears from this study that keeping the line voltage profile requirement (which is a basic criterion for connecting a unit in the Czech Republic) is not suitable for all situations, especially where there are weakly supplied parts of the network. An earlier study proved that losses can be reduced due to the voltage profile after connecting the distributed generators. However, new problems with stability arise.

Simulations were performed using Simulink in order to analyze the voltage stability of the synchronous generators during the mechanical power oscillations that represent wind speed changes. These oscillations are simulated by a sudden step of synchronous generator torque, as shown in Fig. 4. This study is made for several cases of compensation, such 


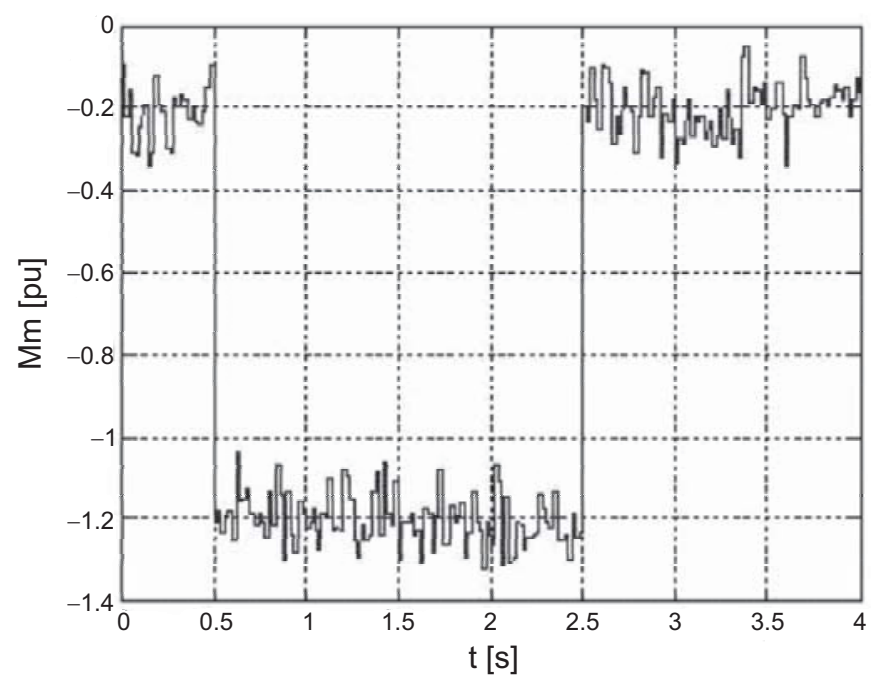

Fig. 4: Wind generator mechanical torque

as compensation of the reactive power of a load and generator, a generator only, or no compensation. In each case the torque step causes a voltage drop, and its stability is shown in Fig. 5, Fig. 6, Fig. 7, Fig. 8 and Fig. 9.

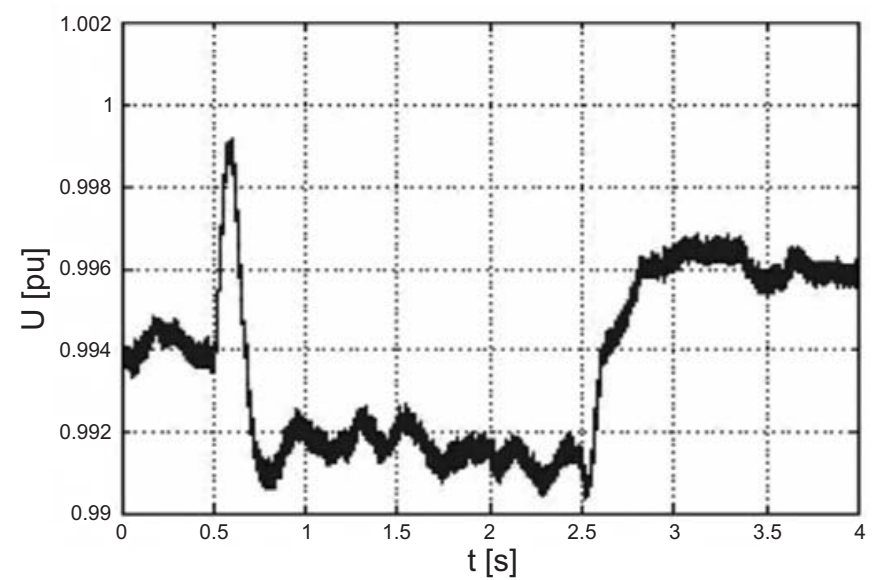

Fig. 5: Voltage: load and generator reactive power compensation - stable case

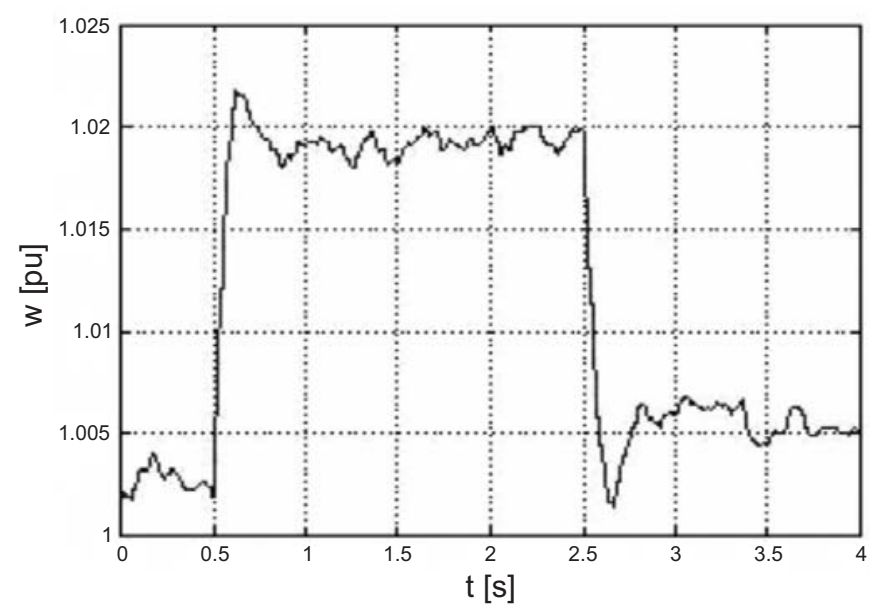

Fig. 6: Rotor speed: load and generator reactive power compensation - stable case

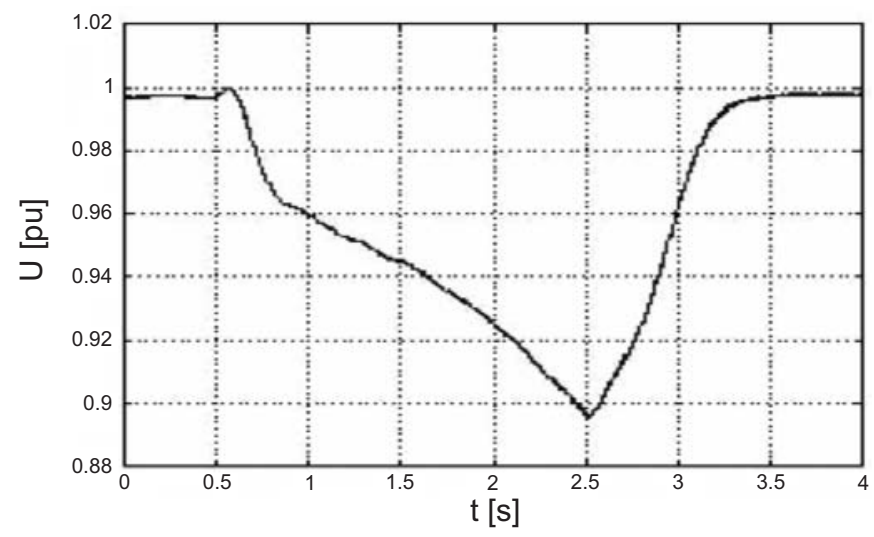

Fig. 7: Voltage: only generator reactive power compensation - unstable case

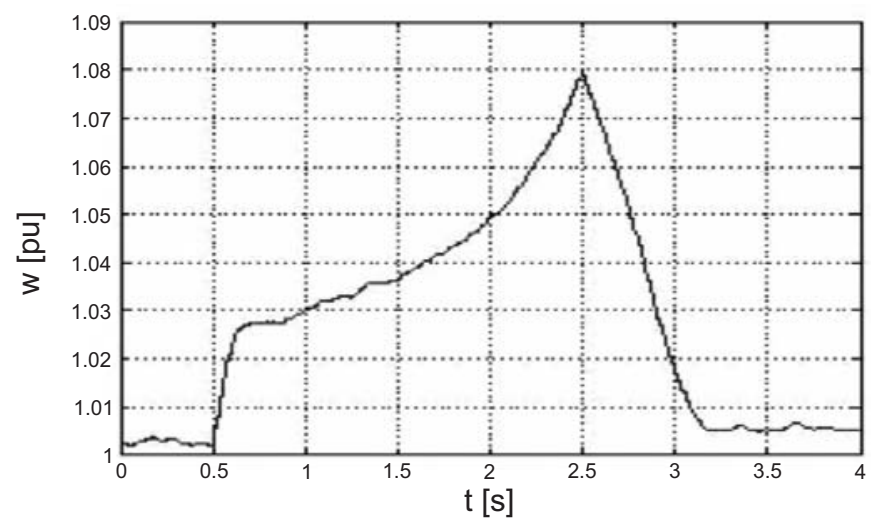

Fig. 8: Rotor speed: only generator reactive power compensation - unstable case

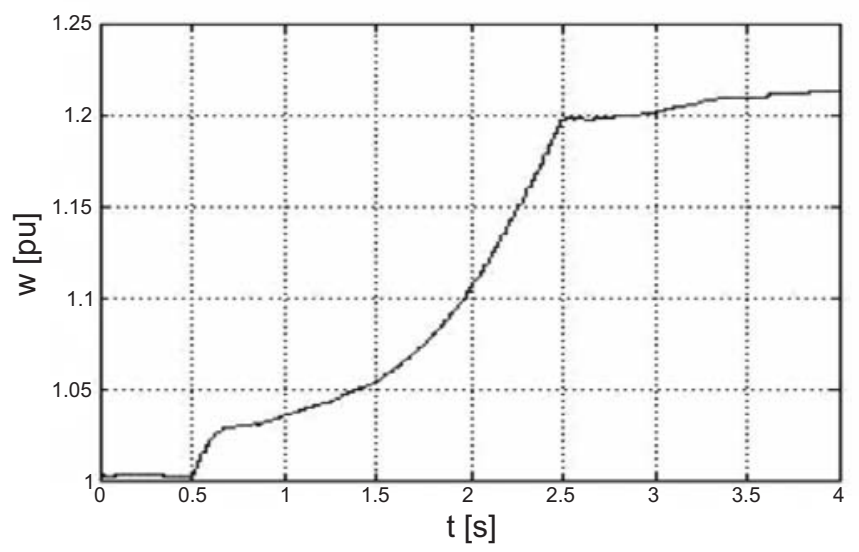

Fig. 9: Rotor speed: no reactive power compensation - unstable case

The first case (load and generator reactive power compensation) is stable, and the voltage drop is negligible. The second case (only generator reactive power compensation) is quasi-stable. A high level of torque causes instability, but when the torque drops, the parameters of the system return to their original state. The stability depends on the time period of the power oscillations. The third case (no compensation) causes high-level instability for the selected duration of the torque.

This study has proved the existence of new criteria for connecting distributed generator units into a distribution net- 
work. Improvements have to be made on both sides, e. g., unit type, construction and regulation and distribution network architecture, control and regulation.

\section{Conclusion}

On the basis of a discussion the general problem of electricity generation from renewable energy sources, more specific ally wind power plants, this paper has described the conditions for connecting these sources to the distribution network. The important part when assessing these plants is the conditions for connection and their negative impacts (voltage change, harmonics, flicker, negative impacts on the ripple control signal) in the distribution system. The paper included a study of connecting a wind power plant to the $22 \mathrm{kV}$ distribution network. The parameters were calculated according to the prescribed rules for connection. The calculation results were analyzed and compared with the conditions for connecting a distributed generation. The study also deals with steady state stability and the impact of the wind power plant on the distribution network.

\section{Acknowledgments}

The research described in this paper was supervised by Prof. J. Tlustý of the Electrical Power Engineering Department, FEE CTU in Prague.

\section{References}

[1] Rules for the operation of distribution systems in Czech Republic.

[2] Kersting, W. H.: Distribution System Modeling and Analysis. CRC Press, 2002.

[3] Sýkora, T.: Rozptýlená výroba elektrické energie (Dispersed Electrical Energy Generation). Diploma thesis, FEE CTU, Prague, 2004.

[4] Czech Standards. Parameters of Power Quality. PNE 333.

Ing. Erick Vincent Mgaya

e-mail:mgayae1@fel.cvut.cz

Ing. Zdeněk Müller

phone: +420 2243352372

fax: +420233337556

e-mail: mullez1@fel.cvut.cz

Department of Electrical Power Engineering

Czech Technical University in Prague

Faculty of Electrical Engineering

Technická 2

16627 Prague 6, Czech Republic 